\title{
Dominance Order and Graphical Partitions
}

\author{
Axel Kohnert \\ Lehrstuhl Mathematik II \\ University of Bayreuth \\ 95440 Bayreuth \\ Germany \\ axel.kohnerteuni-bayreuth.de
}

Submitted: Mar 5, 2003; Accepted: Aug 18, 2003; Published: Mar 5, 2004.

MR Subject Classifications:05A17, 05C07

\begin{abstract}
We gave a new criterion for graphical partitions. We derive a new recursion formula, which allows the computation of the number $g(n)$ of graphical partitions of weight $n$ for up to $n>900$.
\end{abstract}

\section{Introduction}

A partition $\lambda$ of weight $n$ is a nonincreasing sequence of nonnegative integers $\left(\lambda_{1}, \lambda_{2}, \ldots\right.$, $\left.\lambda_{k}, \ldots\right)$ whose sum is $n$. The weight is denoted by $|\lambda|$. The number of nonzero elements in the sequence is the length of the partition denoted by $l(\lambda)$. The set of all partitions of weight $n$ is denoted by $P(n)$. The number of partitions of weight $n$ is denoted by $p(n)$. There is one partition of weight 0 , it is the partition of length 0 . A partition is called graphical if it is the degree sequence of an undirected simple graph. As each edge is the graph is counted twice, a graphical partition must be of even weight. The partition of weight 0 is graphical as it corresponds to a graph without edges. The set of graphical partitions of weight $n$ is denoted by $G(n)$. The number of graphical partitions is denoted by $g(n)$.

A partition $\lambda$ is visualized using the Ferrer's diagram $F_{\lambda}$, i.e. an array of $\lambda_{i}$ left justified boxes in the i-th row of the first quadrant of the plane. The number of boxes on the main diagonal of the Ferrer's diagram $F_{\lambda}$ is the Durfee size of the partition and denoted by $d(\lambda)$. The subpartition built from the $d(\lambda) \times d(\lambda)$ boxes is called the Durfee square of the partition $\lambda$. If we count the number of boxes in each column of the Ferrer's diagram $F_{\lambda}$, we get again a partition, which is 
called the conjugate partition and is denoted by $\lambda^{\prime}$. There are several partial orders on the set of all partitions. We are interested in the dominance order. A partition $\lambda$ is dominated by the partition $\mu$, denoted by $\lambda \unlhd \mu$ if $\sum_{i=1}^{k} \lambda_{i} \leq \sum_{i=1}^{k} \mu_{i}$ for all $k>0$. This is a partial order, as there are pairs of partitions which are not comparable. (e.g. $(5,1,1,1,1,1)$ and $(2,2,2,2,2)$ ).

\section{Criterion}

It is possible to write a partition $\lambda$ as a unique tuple of 3 smaller partitions using a decomposition according to the Durfee square. The first partition $L(\lambda)$ is defined to be

$$
\left(\lambda_{1}^{\prime}-d(\lambda), \ldots, \lambda_{d(\lambda)}^{\prime}-d(\lambda)\right)
$$

The second one $M(\lambda)$ is the Durfee square minus one column. The third one $R(\lambda)$ is defined to be

$$
\left(\lambda_{1}-d(\lambda)+1, \ldots, \lambda_{d(\lambda)}-d(\lambda)+1\right) .
$$

In the following figure these three partitions are marked with $\mathrm{R}, \mathrm{M}$ and $\mathrm{L}$.

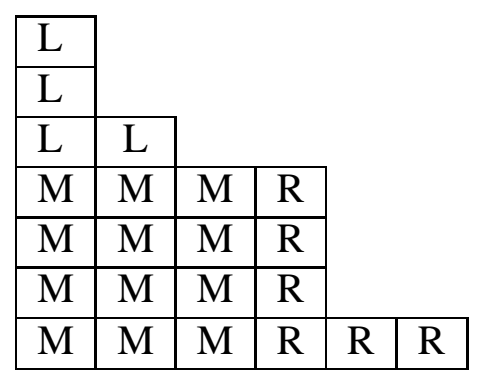

The three corresponding partitions are $L((6,4,4,4,2,1))=(3,1), M((6,4,4,4,2,1))=(3,3,3,3)$ and $R((6,4,4,4,2,1))=(3,1,1,1)$. Now it is possible to give a theorem which connects the question of being graphic with the dominance order of partitions.

Theorem 1 A partition $\lambda$ is graphical if and only if

$$
L(\lambda) \unrhd R(\lambda)
$$

This is a corollary of the criterion of Hässelbarth [Ha] which says that $\lambda$ is graphical if and only if

$$
\sum_{i=1}^{r}\left(\lambda_{i}-\lambda_{i}^{\prime}+1\right) \leq 0
$$

for all $1 \leq r \leq d(\lambda)$ 


\section{Recurrence}

Using the above criterion it is possible to compute the number of graphical partitions of weight $n$. We define

$$
G(n)=: G_{1}(n) \cup \ldots \cup G_{d}(n)
$$

where $G_{i}(n)$ is the set of graphical partitions of weight $n$ and a Durfee square of size $i$. To get the partitions in $G_{i}(n)$ we have to get all pairs of possible $L(\lambda)$ and $R(\lambda)$. The criterion provides a bijection

$$
G_{i}(n) \rightarrow\{(\mu, \nu) \text { with } \mu \unrhd \nu, l(\mu) \leq i, l(\nu)=i,|\nu|+|\mu|=n-(i-1) * i\} .
$$

We denote by $g_{i}(n)$ the number of partitions in $G_{i}(n)$. The set on the right hand side will be decomposed into smaller subsets well suited for the recursion. We denote by $P(m, k, n, l)$ the set of pairs of partitions $(\mu, \nu)$ with the following properties

- $\mu$ is a partition of weight $m$ with length $k$

- $\nu$ is a partition of weight $n$ with length $l$

- $\mu \unrhd \nu$

So we get a bijection with $r=n-i *(i-1)$

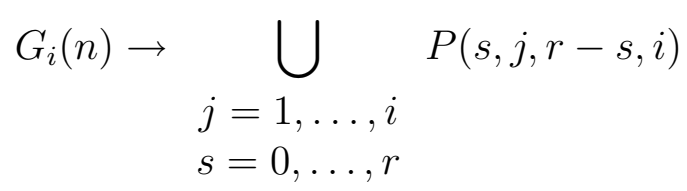

Defining $p(m, k, n, l)$ to be the order of $P(m, k, n, l)$ we use this bijection for the computation of $g(n)$. As we are only interested in the number of partitions we get:

$$
g_{i}(n)=\sum_{\substack{j=1, \ldots, i \\ s=0, \ldots, r}} p(s, j, r-s, i)
$$

Last step in the algorithm for the computation of the number of graphical partitions is the following bijection:

\section{Lemma 2}

$$
\begin{aligned}
P(m, k, n, l) \rightarrow & \bigcup \begin{array}{l}
i=0, \ldots, k \\
j=0, \ldots, l
\end{array} \\
&
\end{aligned}
$$

is a bijection given by removal/addition of the first column in the pair of partitions. 
Proof:

Take a pair $(L, R)$ of partitions from $P(m, k, n, l)$, so for example $L=(3,2,2), R=(3,1,1,1)$ :

\begin{tabular}{|c|c|c|c|c|c|}
\hline $\mathrm{L}$ & & & & & \\
\hline $\mathrm{L}$ & $\mathrm{L}$ & $\mathrm{L}$ & & & \\
\hline $\mathrm{L}$ & $\bar{L}$ & $\overline{\mathrm{L}}$ & & & \\
\hline $\mathrm{M}$ & $\overline{\mathrm{M}}$ & $M$ & $\overline{\mathrm{R}}$ & & \\
\hline $\mathrm{M}$ & $\overline{\mathrm{M}}$ & $\bar{M}$ & $\mathrm{R}$ & & \\
\hline $\mathrm{M}$ & $\bar{M}$ & $\mathrm{M}$ & $\mathrm{R}$ & & \\
\hline $\mathrm{M}$ & $\overline{\mathrm{M}}$ & $\mathrm{M}$ & $\mathrm{R}$ & $\mathrm{R}$ & $\mathrm{R}$ \\
\hline
\end{tabular}

We remove in the Ferrer's diagrams the first column of $L$ and $R$, and get a pair of partitions $\hat{L}, \hat{R}$ of smaller weight:

\begin{tabular}{|c|c|c|c|c|}
\hline $\bar{L}$ & & & & \\
\hline$\hat{L}$ & $\hat{L}$ & $\hat{L}$ & & \\
\hline $\bar{M}$ & $\bar{M}$ & $\bar{M}$ & & \\
\hline $\bar{M}$ & $\mathrm{M}$ & $\mathrm{M}$ & & \\
\hline $\bar{M}$ & $\bar{M}$ & $\overline{\mathrm{M}}$ & & \\
\hline $\bar{M}$ & $\mathrm{M}$ & $\mathrm{M}$ & $\hat{R}$ & $\hat{R}$ \\
\hline
\end{tabular}

When we check the different cases for the lengths of the partitions $L$ and $R$ we get $\hat{L} \unrhd \hat{R}$, so the pair $\hat{L}, \hat{R}$ is element of $P(m-k, i, n-l, j)$ with $i=$ length of the second column of $L$, and $j=$ length of the second column of $R$.

Using this recursion on

$$
p(m, k, n, l)=\sum_{\substack{i=0, \ldots, k \\ j=0, \ldots, l}} p(m-k, i, n-l, j)
$$

we computed recursively the number of graphical partitions.

\subsection{Properties of $p(m, k, n, l)$}

There are several properties of the values $p(m, k, n, l)$ which allow the faster computation of the number of graphical partitions. We have to count the number of pairs of partitions $(\mu, \nu)$. There is a unique lexicographical minimal partition $\mu_{-}$with weight $m$ and length $k$, and a unique lexicographical maximal partition $\nu^{+}$with weight $n$ and length $l$. We have the following lemma 
Lemma 3 For $m, k, n, l$ with $\mu_{-} \unrhd \nu^{+}$we have $p(m, k, n, l)=p(m, k, 0,0) p(n, l, 0,0)$.

as every partition in the set $P(m, k):=P(m, k, 0,0)$ dominates any partition from $P(n, l)$.

In the case of $m>n$ we get a telescoping sum (thanks to the referee) which allows a fast computation in this case

Lemma 4 For $m>n$ we have:

$$
\begin{aligned}
p(m, k, n, l)= & p(m-1, k-1, n, l)+p(m, k, n-1, l-1) \\
& -p(m-1, k-1, n-1, l-1)+p(m-k, k, n-l, l) .
\end{aligned}
$$

Proof: start with the difference of

$$
p(m, k, n, l)=\sum_{\substack{i=0, \ldots, k \\ j=0, \ldots, l}} p(m-k, i, n-l, j)
$$

and

$$
p(m-1, k-1, n, l)=\sum_{\substack{i=0, \ldots, k-1 \\ j=0, \ldots, l}} p(m-k, i, n-l, j)
$$

giving

$$
p(m, k, n, l)-p(m-1, k-1, n, l)=\sum_{j=0, \ldots, l} p(m-k, k, n-l, j)
$$

and in the case of $n-1, l-1$ :

$$
p(m, k, n-1, l-1)-p(m-1, k-1, n-1, l-1)=\sum_{j=0, \ldots, l-1} p(m-k, k, n-l, j) .
$$

The difference of the last two lines gives the statement of the lemma.

\section{Computation of $g(n)$}

Like in the case of Barnes and Savage $[\overline{\mathrm{BS}}]$ it is useful to store the computed values $p(m, k, n, l)$ in a four dimensional table. As $k$ and $l$ are limited by $\lfloor\sqrt{n}\rfloor$ the space requirement of the algorithm is like in their case $O\left(n^{3}\right)$. The telescoping lemma helps to speed up the computation, but it does not reduce the amount of memory necessary to store the intermediate results. The formula for the computation of the number of graphical partitions of weight $n$ computes in the outer loop the number of graphical partitions of $n$ with a fixed Durfee size $d$. These numbers, which add up to $g(n)$ are listed in the following table. 


\begin{tabular}{|c|r|r|r|r|r|r|}
\hline $\mathrm{n} / \mathrm{d}$ & 1 & 2 & 3 & 4 & 5 & 6 \\
\hline 2 & 1 & & & & & \\
\hline 4 & 2 & & & & & \\
\hline 6 & 3 & 2 & & & & \\
\hline 8 & 4 & 5 & & & & \\
\hline 10 & 5 & 12 & & & & \\
\hline 12 & 6 & 22 & 3 & & & \\
\hline 14 & 7 & 38 & 9 & & & \\
\hline 16 & 8 & 58 & 24 & & & \\
\hline 18 & 9 & 87 & 55 & & & \\
\hline 20 & 10 & 121 & 108 & 5 & & \\
\hline 22 & 11 & 166 & 195 & 15 & & \\
\hline 24 & 12 & 218 & 335 & 42 & & \\
\hline 26 & 13 & 283 & 539 & 98 & & \\
\hline 28 & 14 & 356 & 832 & 218 & & \\
\hline 30 & 15 & 445 & 1247 & 422 & 7 & \\
\hline 32 & 16 & 543 & 1803 & 788 & 23 & \\
\hline 34 & 17 & 659 & 2542 & 1374 & 65 & \\
\hline 36 & 18 & 786 & 3515 & 2322 & 158 & \\
\hline 38 & 19 & 933 & 4752 & 3743 & 356 & \\
\hline 40 & 20 & 1092 & 6315 & 5881 & 740 & \\
\hline 42 & 21 & 1274 & 8278 & 8931 & 1441 & 11 \\
\hline 44 & 22 & 1469 & 10683 & 13318 & 2653 & 34 \\
\hline 46 & 23 & 1689 & 13619 & 19339 & 4699 & 98 \\
\hline 48 & 24 & 1924 & 17182 & 27612 & 8016 & 238 \\
\hline 50 & 25 & 2186 & 21435 & 38656 & 13257 & 545 \\
\hline 52 & 26 & 2464 & 26490 & 53412 & 21267 & 1143 \\
\hline
\end{tabular}

We computed the number of graphical partitions up to $n>900$. This table extends the table previously published by Barnes and Savage $[\mathrm{BS}]$. Like in their table we include $p(n)$ the number of partitions and the ratio $g(n) / p(n)$. 


\begin{tabular}{|c|c|c|c|c|c|c|c|}
\hline$n$ & $g(n)$ & $p(n)$ & $g(n) / p(n)$ & $n$ & $g(n)$ & $p(n)$ & $g(n) / p(n)$ \\
\hline 2 & 1 & 2 & .5000000 & 58 & 264941 & 715220 & .3704328 \\
\hline 4 & 2 & 5 & .4000000 & 60 & 357635 & 966467 & .3700436 \\
\hline 6 & 5 & 11 & .4545454 & 62 & 480408 & 1300156 & .3695002 \\
\hline 8 & 9 & 22 & .4090909 & 64 & 642723 & 1741630 & .3690353 \\
\hline 10 & 17 & 42 & .4047619 & 66 & 856398 & 2323520 & .3685778 \\
\hline 12 & 31 & 77 & .4025974 & 68 & 1136715 & 3087735 & .3681387 \\
\hline 14 & 54 & 135 & .4000000 & 70 & 1503172 & 4087968 & .3677064 \\
\hline 16 & 90 & 231 & .3896103 & 72 & 1980785 & 5392783 & .3673029 \\
\hline 18 & 151 & 385 & .3922077 & 74 & 2601057 & 7089500 & .3668886 \\
\hline 20 & 244 & 627 & .3891547 & 76 & 3404301 & 9289091 & .3664837 \\
\hline 22 & 387 & 1002 & .3862275 & 78 & 4441779 & 12132164 & .3661159 \\
\hline 24 & 607 & 1575 & .3853968 & 80 & 5777292 & 15796476 & .3657329 \\
\hline 26 & 933 & 2436 & .3830049 & 82 & 7492373 & 20506255 & .3653701 \\
\hline 28 & 1420 & 3718 & .3819257 & 84 & 9688780 & 26543660 & .3650129 \\
\hline 30 & 2136 & 5604 & .3811563 & 86 & 12494653 & 34262962 & .3646693 \\
\hline 32 & 3173 & 8349 & .3800455 & 88 & 16069159 & 44108109 & .3643130 \\
\hline 34 & 4657 & 12310 & .3783103 & 90 & 20614755 & 56634173 & .3639985 \\
\hline 36 & 6799 & 17977 & .3782054 & 92 & 26377657 & 72533807 & .3636601 \\
\hline 38 & 9803 & 26015 & .3768210 & 94 & 33671320 & 92669720 & .3633475 \\
\hline 40 & 14048 & 37338 & .3762386 & 96 & 42878858 & 118114304 & .3630284 \\
\hline 42 & 19956 & 53174 & .3752961 & 98 & 54481054 & 150198136 & .3627278 \\
\hline 44 & 28179 & 75175 & .3748453 & 100 & 69065657 & 190569292 & .3624175 \\
\hline 46 & 39467 & 105558 & .3738892 & 102 & 87370195 & 241265379 & .3621331 \\
\hline 48 & 54996 & 147273 & .3734289 & 104 & 110287904 & 304801365 & .3618353 \\
\hline 50 & 76104 & 204226 & .3726459 & 106 & 138937246 & 384276336 & .3615555 \\
\hline 52 & 104802 & 281589 & .3721807 & 108 & 174675809 & 483502844 & .3612715 \\
\hline 54 & 143481 & 386155 & .3715632 & 110 & 219186741 & 607163746 & .3610010 \\
\hline 56 & 195485 & 526823 & .3710639 & 112 & 274512656 & 761002156 & .3607252 \\
\hline
\end{tabular}


To group digits in the larger numbers we included a '.' at every sixth position.

\begin{tabular}{|c|c|c|c|}
\hline$n$ & $g(n)$ & $p(n)$ & $g(n) / p(n)$ \\
\hline 114 & 343181668 & 952050665 & .3604657 \\
\hline 116 & 428244215 & 1188908248 & .3601995 \\
\hline 118 & 533464959 & 1482074143 & .3599448 \\
\hline 120 & 663394137 & 1844349560 & .3596900 \\
\hline 122 & 823598382 & 2291.320912 & 3594426 \\
\hline 124 & 1020807584 & 2841.940500 & 3591938 \\
\hline 126 & 1263243192 & 3519.222692 & .3589551 \\
\hline 128 & 1560795436 & 4351.078600 & .3587146 \\
\hline 130 & 1925513465 & 5371.315400 & .3584808 \\
\hline 132 & 2371.901882 & 6620.830889 & .3582483 \\
\hline 134 & 2917.523822 & 8149.040695 & .3580205 \\
\hline 136 & 3583.515700 & 10015.581680 & 3577940 \\
\hline 138 & 4395.408234 & 12292.341831 & .3575728 \\
\hline 140 & 5383.833857 & 15065.878135 & .3573528 \\
\hline 142 & 6585.699894 & 18440.293320 & .3571363 \\
\hline 144 & 8045.274746 & 22540.654445 & 3569228 \\
\hline 146 & 9815.656018 & 27517.052599 & 3567117 \\
\hline 148 & 11960.467332 & 33549.419497 & .3565029 \\
\hline 150 & 14555.902348 & 40853.235313 & .3562974 \\
\hline 152 & 17692.990183 & 49686.288421 & 3560940 \\
\hline 154 & 21480.510518 & 60356.673280 & 3558928 \\
\hline 156 & 26048.320019 & 73232.243759 & .3556946 \\
\hline 158 & 31551.087790 & 88751.778802 & 3554980 \\
\hline 160 & 38173.235010 & 107438.159466 & .3553042 \\
\hline 162 & 46134.037871 & 129913.904637 & .3551123 \\
\hline 164 & 55694.314567 & 156919.475295 & 3549228 \\
\hline 166 & 67163.674478 & 189334.822579 & .3547349 \\
\hline 168 & 80909.973315 & 228204.732751 & .3545499 \\
\hline 170 & 97368.672089 & 274768.617130 & .3543660 \\
\hline 172 & 117056.456152 & 330495.499613 & .3541847 \\
\hline 174 & 140584.220188 & 397125.074750 & .3540048 \\
\hline 176 & 168675.124141 & 476715.857290 & .3538273 \\
\hline 178 & 202182.888436 & 571701.605655 & .3536510 \\
\hline 180 & 242116.891036 & 684957.390936 & .3534773 \\
\hline 182 & 289666.252014 & 819876.908323 & .3533045 \\
\hline 184 & 346234.896845 & 980462.880430 & .3531341 \\
\hline 186 & 413474.657328 & 1.171432 .692373 & .3529649 \\
\hline 188 & 493331.835384 & 1.398341 .745571 & .3527977 \\
\hline 190 & 588093.594457 & 1.667727 .404093 & .3526317 \\
\hline
\end{tabular}




\begin{tabular}{|c|c|c|c|}
\hline$n$ & $g(n)$ & $p(n)$ & $g(n) / p(n)$ \\
\hline 192 & 700451.190712 & 1.987276 .856363 & 3524678 \\
\hline 194 & 833561.537987 & 2.366022 .741845 & .3523049 \\
\hline 196 & 991134.281267 & 2.814570 .987591 & 3521439 \\
\hline 198 & 1.177516 .049387 & 3.345365 .983698 & 3519842 \\
\hline 200 & 1.397805 .210533 & 3.972999 .029388 & .3518262 \\
\hline 202 & 1.657968 .320899 & 4.714566 .886083 & .3516692 \\
\hline 204 & 1.964994 .991232 & 5.590088 .317495 & .3515141 \\
\hline 206 & 2.327052 .859551 & 6.622987 .708040 & .3513599 \\
\hline 208 & 2.753697 .110356 & 7.840656 .226137 & .3512074 \\
\hline 210 & 3.256081 .386335 & 9.275102 .575355 & 3510561 \\
\hline 212 & 3.847232 .865612 & 10.963707 .205259 & .3509062 \\
\hline 214 & 4.542341 .563460 & 12.950095 .925895 & .3507573 \\
\hline 216 & 5.359127 .512113 & 15.285151 .248481 & .3506100 \\
\hline 218 & 6.318223 .879596 & 18.028182 .516671 & .3504637 \\
\hline 220 & 7.443670 .977177 & 21.248279 .009367 & 3503187 \\
\hline 222 & 8.763432 .946593 & 25.025873 .760111 & .3501749 \\
\hline 224 & 10.310044 .123494 & 29.454549 .941750 & .3500323 \\
\hline 226 & 12.121309 .266199 & 34.643126 .322519 & 3498907 \\
\hline 228 & 14.241160 .856051 & 40.718063 .627362 & .3497504 \\
\hline 230 & 16.720586 .202163 & 47.826239 .745920 & 3496111 \\
\hline 232 & 19.618767.868192 & 56.138148 .670947 & .3494730 \\
\hline 234 & 23.004324 .059046 & 65.851585 .970275 & .3493359 \\
\hline 236 & 26.956798 .814985 & 77.195892 .663512 & 3491999 \\
\hline 238 & 31.568326 .350604 & 90.436839 .668817 & 3490648 \\
\hline 240 & 36.945596 .125431 & 105.882246 .722733 & 3489309 \\
\hline 242 & 43.212042 .821600 & 123.888443 .077259 & 3487980 \\
\hline 244 & 50.510448 .519684 & 144.867692 .496445 & .3486660 \\
\hline 246 & 59.005849 .206367 & 169.296722 .391554 & .3485350 \\
\hline 248 & 68.888924 .114697 & 197.726516 .681672 & .3484050 \\
\hline 250 & 80.379859 .814364 & 230.793554 .364681 & .3482760 \\
\hline 252 & 93.732799 .789716 & 269.232701 .252579 & 3481479 \\
\hline 254 & 109.240907.229098 & 313.891991 .306665 & 3480206 \\
\hline 256 & 127.242219 .898679 & 365.749566 .870782 & 3478943 \\
\hline 258 & 148.126317 .233645 & 425.933084 .409356 & .3477689 \\
\hline 260 & 172.341932 .589627 & 495.741934 .760846 & .3476444 \\
\hline 262 & 200.405745 .147874 & 576.672674 .947168 & .3475207 \\
\hline 264 & 232.912328 .227060 & 670.448123 .060170 & 3473979 \\
\hline
\end{tabular}




\begin{tabular}{|c|c|c|c|}
\hline$n$ & $g(n)$ & $p(n)$ & $g(n) / p(n)$ \\
\hline 266 & 270.545608 .772217 & 779.050629 .562167 & 3472760 \\
\hline 268 & 314.091890 .030723 & 904.760108 .316360 & 3471548 \\
\hline 270 & 364.454850 .689480 & 1050.197489 .931117 & 3470345 \\
\hline 272 & 422.672418 .723168 & 1218.374349 .844333 & 3469150 \\
\hline 274 & 489.936412 .910522 & 1412.749565 .173450 & 3467963 \\
\hline 276 & 567.614507 .770134 & 1637.293969 .337171 & 3466784 \\
\hline 278 & 657.275703 .933020 & 1896.564103 .591584 & 3465612 \\
\hline 280 & 760.718950 .656347 & 2195.786311 .682516 & 3464448 \\
\hline 282 & 880.006264 .424357 & 2540.952590 .045698 & 3463292 \\
\hline 284 & 1017.499729 .851133 & 2938.929793 .929555 & .3462143 \\
\hline 286 & 1175.904589 .041542 & 3397.584011 .986773 & 3461002 \\
\hline 288 & 1358.317187 .000975 & 3925.922161 .489422 & .3459867 \\
\hline 290 & 1568.280617 .221370 & 4534.253126 .900886 & 3458740 \\
\hline 292 & 1809.846889 .359039 & 5234.371069 .753672 & 3457620 \\
\hline 294 & 2087.648920 .451849 & 6039.763882 .095515 & 3456507 \\
\hline 296 & 2406.980630 .541347 & 6965.850144 .195831 & 3455401 \\
\hline 298 & 2773.890059 .176591 & 8030.248384 .943040 & 3454301 \\
\hline 300 & 3195.282761 .990490 & 9253.082936 .723602 & .3453208 \\
\hline 302 & 3679.041523 .618584 & 10657.331232 .548839 & 3452122 \\
\hline 304 & 4234.159847 .629493 & 12269.218019 .229465 & .3451042 \\
\hline 306 & 4870.896069 .907545 & 14118.662665 .280005 & .3449969 \\
\hline 308 & 5600.944751 .183391 & 16239.786535 .829663 & 3448902 \\
\hline 310 & 6437.635040 .483873 & 18671.488299 .600364 & 3447842 \\
\hline 312 & 7396.150995 .787149 & 21458.096037 .352891 & 3446788 \\
\hline 314 & 8493.785631 .612121 & 24650.106150 .830490 & 3445739 \\
\hline 316 & 9750.224120 .415064 & 28305.020340 .996003 & .3444697 \\
\hline 318 & 11187.869357 .515526 & 32488.293351 .466654 & 3443661 \\
\hline 320 & 12832.204376 .370829 & 37274.405776 .748077 & 3442631 \\
\hline 322 & 14712.209437 .460953 & 42748.078035 .954696 & 3441607 \\
\hline 324 & 16860.826086 .379188 & 49005.643635 .237875 & .3440588 \\
\hline 326 & 19315.489319 .698561 & 56156.602112 .874289 & .3439575 \\
\hline 328 & 22118.721571 .923434 & 64325.374609 .114550 & 3438568 \\
\hline 330 & 25318.812013 .118277 & 73653.287861 .850339 & 3437567 \\
\hline 332 & 28970.573903 .861784 & 84300.815636 .225119 & 3436571 \\
\hline 334 & 33136.211302 .816850 & 96450.110192 .202760 & .3435580 \\
\hline 336 & 37886.285108 .888194 & 110307.860425 .292772 & .3434595 \\
\hline 338 & 43300.814969 .998852 & 126108.517833 .796355 & .3433615 \\
\hline
\end{tabular}




\begin{tabular}{|c|c|c|c|}
\hline$n$ & $g(n)$ & $p(n)$ & $g(n) / p(n)$ \\
\hline 340 & 49470.510879 .271020 & 144117.936527 .873832 & 3432640 \\
\hline 342 & 56498.174296 .996950 & 164637.479165 .761044 & .3431671 \\
\hline 344 & 64500.263250 .927647 & 188008.647052 .292980 & .3430707 \\
\hline 346 & 73608.673091 .026153 & 214618.299743.286299 & 3429748 \\
\hline 348 & 83972.725212 .921328 & 244904.537455 .382406 & .3428794 \\
\hline 350 & 95761.423661 .105714 & 279363.328483 .702152 & .3427845 \\
\hline 352 & 109165.980705.294681 & 318555.973788 .329084 & .3426901 \\
\hline 354 & 124402.676259 .476568 & 363117.512048 .110005 & .3425961 \\
\hline 356 & 141716.056306 .961362 & 413766.180933 .342362 & .3425027 \\
\hline 358 & 161382.553841 .124116 & 471314.064268 .398780 & 3424097 \\
\hline 360 & 183714.538700 .727580 & 536679.070310 .691121 & .3423173 \\
\hline 362 & 209064.891369 .055649 & 610898.403751 .884101 & .3422253 \\
\hline 364 & 237832.127202 .308431 & 695143.713458 .946040 & .3421337 \\
\hline 366 & 270466.172555 .733767 & 790738.119649 .411319 & .3420426 \\
\hline 368 & 307474.832766 .566116 & 899175.348396 .088349 & .3419520 \\
\hline 370 & 349431.082262 .461473 & 1.022141 .228367 .345362 & 3418618 \\
\hline 372 & 396981.225388 .693589 & 1.161537 .834849 .962850 & .3417721 \\
\hline 374 & 450854.077011 .727696 & 1.319510 .599727 .473500 & .3416828 \\
\hline 376 & 511871.252448 .667437 & 1.498478 .743590 .581081 & .3415939 \\
\hline 378 & 580958.724595 .080633 & 1.701169 .427975 .813525 & .3415055 \\
\hline 380 & 659159.770630 .009034 & 1.930656 .072350 .465812 & 3414175 \\
\hline 382 & 747649.510890 .816168 & 2.190401 .332423 .765131 & 3413299 \\
\hline 384 & 847751.191197 .762717 & 2.484305 .294265 .418180 & 3412427 \\
\hline 386 & 960954.438051 .593305 & 2.816759.503217.942792 & 3411560 \\
\hline 388 & 1.088935 .719745 .607833 & 3.192707 .518433 .532826 & .3410696 \\
\hline 390 & 1.233581 .257527 .175008 & 3.617712 .763867 .604423 & 3409837 \\
\hline 392 & 1.397012 .690532 .962312 & 4.098034.535626.594791 & 3408982 \\
\hline 394 & 1.581615 .810583 .814123 & 4.640713 .124699 .623515 & .3408130 \\
\hline 396 & 1.790072 .736673 .606208 & 5.253665 .124416 .975163 & .3407283 \\
\hline 398 & 2.025397 .891141 .779483 & 5.945790 .114707 .874597 & .3406440 \\
\hline 400 & 2.290978 .305361 .293873 & 6.727090 .051741 .041926 & .3405600 \\
\hline 402 & 2.590618 .639582 .366767 & 7.608802 .843339 .879269 & .3404765 \\
\hline 404 & 2.928591 .589058 .407676 & 8.603551 .759348 .655060 & .3403933 \\
\hline 406 & 3.309694 .180956 .900829 & 9.725512 .513742 .021729 & .3403105 \\
\hline 408 & 3.739310 .775529 .786273 & 10.990600 .063775 .926994 & 3402280 \\
\hline 410 & 4.223483 .357738 .533049 & 12.416677 .403151 .190382 & .3401460 \\
\hline 412 & 4.768990 .219275 .581506 & 14.023788 .883518 .847344 & .3400643 \\
\hline
\end{tabular}




\begin{tabular}{|c|c|c|c|}
\hline$n$ & $g(n)$ & $p(n)$ & $g(n) / p(n)$ \\
\hline 414 & 5.383433 .672948 .398244 & 15.834420 .884488 .187770 & 3399829 \\
\hline 416 & 6.075338 .174381 .850575 & 17.873792 .969689 .876004 & 3399020 \\
\hline 418 & 6.854259 .698107 .669457 & 20.170183 .018805 .933659 & 3398213 \\
\hline 420 & 7.730908 .023798 .421590 & 22.755290 .216580 .025259 & 3397411 \\
\hline 422 & 8.717282 .933540 .859360 & 25.664640 .213837 .714846 & 3396612 \\
\hline 424 & 9.826826 .500401 .651249 & 28.938037 .257084 .798150 & .3395816 \\
\hline 426 & 11.074592 .583508 .500810 & 32.620068 .617410 .232189 & .3395024 \\
\hline 428 & 12.477436 .219213 .567055 & 36.760667 .241831 .527309 & 3394235 \\
\hline 430 & 14.054224 .400690 .717799 & 41.415739 .207102 .358378 & 3393450 \\
\hline 432 & 15.826071 .462575 .276026 & 46.647863 .284229 .267991 & 3392668 \\
\hline 434 & 17.816600 .870069 .735539 & 52.527070 .729108 .240605 & 3391889 \\
\hline 436 & 20.052237 .544857 .629067 & 59.131714 .309169 .618645 & 3391113 \\
\hline 438 & 22.562532 .800060 .770285 & 66.549436 .566966 .297367 & 3390341 \\
\hline 440 & 25.380526 .940366 .383372 & 74.878248 .419470 .886233 & 3389572 \\
\hline 442 & 28.543152 .282052 .209031 & 84.227730 .407729 .499781 & .3388807 \\
\hline 444 & 32.091682 .620530 .574852 & 94.720370 .257893 .471820 & 3388044 \\
\hline 446 & 36.072232 .518846 .606193 & 106.493051 .905239 .118581 & .3387285 \\
\hline 448 & 40.536314 .045019 .830519 & 119.698712 .782720 .205954 & .3386528 \\
\hline 450 & 45.541454 .927569 .937980 & 134.508188 .001572 .923840 & .3385775 \\
\hline 452 & 51.151887 .377895 .518187 & 151.112262 .071917 .313678 & .3385025 \\
\hline 454 & 57.439312 .856875 .164198 & 169.723951 .046458 .040965 & .3384278 \\
\hline 456 & 64.483753 .765628 .119990 & 190.581040 .442651 .931034 & .3383534 \\
\hline 458 & 72.374498 .575459 .529761 & 213.948907 .032733 .069132 & .3382793 \\
\hline 460 & 81.211154 .126223 .919812 & 240.123655 .613925 .192081 & 3382055 \\
\hline 462 & 91.104812 .889042 .494402 & 269.435605 .212954 .994471 & 3381320 \\
\hline 464 & 102.179351 .774084 .517247 & 302.253162 .872576 .636605 & 3380588 \\
\hline 466 & 114.572872 .711521 .982305 & 338.987127 .249525 .432549 & 3379859 \\
\hline 468 & 128.439304 .643242 .346397 & 380.095468 .763120 .598477 & 3379132 \\
\hline 470 & 143.950179 .632789 .523790 & 426.088638 .015652 .413417 & 3378409 \\
\hline 472 & 161.296607 .420846 .627003 & 477.535459 .708164 .115593 & 3377688 \\
\hline 474 & 180.691463 .780462 .230570 & 535.069675 .351607 .262125 & 3376970 \\
\hline 476 & 202.371821 .906641 .398466 & 599.397204 .782301 .852926 & 3376255 \\
\hline 478 & 226.601646 .793972 .364092 & 671.304203 .896731 .807232 & .3375543 \\
\hline 480 & 253.674787 .206902 .086847 & 751.666004 .194993 .125591 & .3374833 \\
\hline 482 & 283.918289 .980520 .154068 & 841.457028 .742823 .649455 & .3374127 \\
\hline 484 & 317.696079 .235783 .042618 & 941.761789 .114997 .698055 & .3373422 \\
\hline 486 & 355.413030 .566947 .436185 & 1053.787078 .862455 .346513 & 3372721 \\
\hline 488 & 397.519491 .261433 .715764 & 1178.875491 .155735 .802646 & 3372022 \\
\hline 490 & 444.516285 .148721 .676016 & 1318.520401 .612270 .233223 & 3371326 \\
\hline
\end{tabular}




\begin{tabular}{|c|c|c|c|}
\hline$n$ & $g(n)$ & $p(n)$ & $g(n) / p(n)$ \\
\hline 492 & 496.960262 .431221 .933377 & 1474.382572 .040363 .953132 & 3370633 \\
\hline 494 & 555.470442 .345519 .505726 & 1648.308547 .066172 .438760 & .3369942 \\
\hline 496 & 620.734822 .524488 .429887 & 1842.351033 .503159 .891466 & .3369253 \\
\hline 498 & 693.517913 .244644 .376036 & 2058.791472 .042884 .901563 & .3368568 \\
\hline 500 & 774.669085 .011428 .835081 & 2300.165032 .574323 .995027 & .3367884 \\
\hline 502 & 865.131803 .384963 .113026 & 2569.288288 .377098 .289281 & .3367204 \\
\hline 504 & 965.953855 .732975 .451076 & 2869.289850 .802400 .662045 & .3366525 \\
\hline 506 & 1078.298661 .059460 .646172 & 3203.644275 .096202 .070012 & .3365850 \\
\hline 508 & 1203.457790 .569809 .977154 & 3576.209579 .998154 .653671 & .3365176 \\
\hline 510 & 1342.864809 .865594 .854721 & 3991.268758 .958164 .118300 & .3364506 \\
\hline 512 & 1498.110595 .470108 .535477 & 4453.575699 .570940 .947378 & .3363837 \\
\hline 514 & 1670.960265 .197692 .006066 & 4968.405970 .488126 .319775 & .3363171 \\
\hline 516 & 1863.371903 .154222 .485007 & 5541.612982 .013113 .936133 & .3362508 \\
\hline 518 & 2077.517250 .840894 .293529 & 6179.690078 .238084 .808000 & .3361846 \\
\hline 520 & 2315.804584 .308779 .792051 & 6889.839175 .409542 .385648 & .3361188 \\
\hline 522 & 2580.903985 .437582 .047712 & 7680.046623 .716094 .332553 & .3360531 \\
\hline 524 & 2875.775270 .499622 .643254 & 8559.167038 .437716 .736150 & .3359877 \\
\hline 526 & 3203.698835 .512775 .321542 & 9537.015921 .990240 .021538 & .3359225 \\
\hline 528 & 3568.309730 .462613 .277337 & 10624.471981 .512075 .020731 & .3358576 \\
\hline 530 & 3973.635279 .920638 .314843 & 11833.590138 .006300 .416410 & .3357928 \\
\hline 532 & 4424.136628 .837685 .204084 & 13177.726323 .474524 .612308 & .3357283 \\
\hline 534 & 4924.754598 .198148 .903956 & 14671.675272 .840783 .232475 & .3356640 \\
\hline 536 & 5480.960303 .823131 .913346 & 16331.822638 .729701 .493803 & .3356000 \\
\hline 538 & 6098.811014 .380095 .758058 & 18176.312890 .390861 .435034 & .3355362 \\
\hline 540 & 6785.011787 .015484 .143370 & 20225.234604 .409151 .266221 & .3354725 \\
\hline 542 & 7546.983460 .661323 .270992 & 22500.824915 .577356 .165493 & .3354091 \\
\hline 544 & 8392.937659 .823034 .254314 & 25027.695072 .821279 .146420 & .3353460 \\
\hline 546 & 9331.959509 .452339 .672600 & 27833.079238 .879849 .385687 & .3352830 \\
\hline 548 & 10374.098842 .503992 .047601 & 30947.108885 .217475 .101876 & .3352202 \\
\hline 550 & 11530.470761 .851201 .437941 & 34403.115367 .205050 .943160 & .3351577 \\
\hline 552 & 12813.366486 .201931 .098406 & 38237.963520 .943177 .237554 & .3350954 \\
\hline 554 & 14236.375525 .907215 .720937 & 42492.419404 .397720 .872600 & .3350333 \\
\hline 556 & 15814.520314 .233192 .845657 & 47211.555614 .160398 .040338 & .3349713 \\
\hline 558 & 17564.404554 .306849 .379544 & 52445.197947 .746313 .627407 & .3349096 \\
\hline 560 & 19504.376630 .425338 .940450 & 58248.417552 .751868 .050007 & 3348481 \\
\hline 562 & 21654.709624 .095907 .037587 & 64682.073111 .542943 .380454 & .3347868 \\
\hline 564 & 24037.799541 .712545 .312555 & 71813.408056 .839596 .203570 & .3347257 \\
\hline
\end{tabular}




\begin{tabular}{|c|c|c|c|}
\hline$n$ & $g(n)$ & $p(n)$ & $g(n) / p(n)$ \\
\hline 566 & 26678.383616 .684151 .091417 & 79716.708303 .343130 .521599 & .3346648 \\
\hline 568 & 29603.780629 .611359 .695197 & 88474.026517 .495817 .981253 & .3346041 \\
\hline 570 & 32844.155485 .585087 .895823 & 98175.979536 .033971 .312388 & .3345437 \\
\hline 572 & 36432.810377 .738654 .758119 & 108922.626189 .067392 .956037 & . 3344834 \\
\hline 574 & 40406.505261 .273864 .035858 & 120824.433490 .320564 .237125 & .3344232 \\
\hline 576 & 44805.810415 .680682 .864354 & 134003.339931 .725153 .597473 & 3343633 \\
\hline 578 & 49675.494379 .015092 .548130 & 148593.925468 .119890 .197615 & .3343036 \\
\hline 580 & 55064.950610 .484813 .034226 & 164744.698707 .340387 .584240 & .3342441 \\
\hline 582 & 61028.666818 .870688 .179003 & 182619.512839 .056823 .919887 & .3341848 \\
\hline 584 & 67626.740980 .425298 .679830 & 202399.122950 .629095 .580175 & .3341256 \\
\hline 586 & 74925.448815 .380684 .522752 & 224282.898599 .046831 .034631 & .3340667 \\
\hline 588 & 82997.867525 .602669 .487564 & 248490.706844 .586261 .413858 & 3340079 \\
\hline 590 & 91924.561526 .362430 .605299 & 275264.982414 .934173 .206642 & 3339493 \\
\hline 592 & 101794.335967 .391354 .819610 & 304873.003269 .975366 .031783 & 3338909 \\
\hline 594 & 112705.064903 .541372 .910902 & 337609.391590 .065169 .560935 & .3338327 \\
\hline 596 & 124764.601060 .696691 .230392 & 373798.862128 .436852 .709430 & .3337746 \\
\hline 598 & 138091.775476 .065977 .362714 & 413799.241966 .727832 .978027 & .3337168 \\
\hline 600 & 152817.495306 .901705 .606360 & 458004.788008 .144308 .553622 & .3336591 \\
\hline 602 & 169085.949733 .393626 .681353 & 506849.831053 .734861 .481872 & .3336016 \\
\hline 604 & 187055.933951 .850393 .240988 & 560812.778053 .476538 .349420 & .3335443 \\
\hline 606 & 206902.303112 .470251 .801908 & 620420.507127 .059714 .307352 & 3334872 \\
\hline 608 & 228817.568177 .684489 .362279 & 686253.193233 .019826 .880477 & .3334302 \\
\hline 610 & 253013.647961.452399.720977 & 758949.605954.969709.105721 & .3333734 \\
\hline 612 & 279723.791649 .781906 .578355 & 839212.924798 .226411 .060795 & .3333168 \\
\hline 614 & 309204.688862 .398100 .317169 & 927817.121679 .723721 .849795 & .3332603 \\
\hline 616 & 341738.784463 .948448 .266796 & 1.025613 .964982 .134990 .453294 & 3332041 \\
\hline 618 & 377636.818464 .771506 .567385 & 1.133540 .704665 .979618 .906662 & 3331479 \\
\hline 620 & 417240.611615 .194905 .255263 & 1.252628 .503530 .795506 .440909 & .3330920 \\
\hline 622 & 460926.121091 .974999 .638124 & 1.384011 .685831 .426958 .558879 & .3330362 \\
\hline 624 & 509106.790873 .918774 .794943 & 1.528937 .881135 .168275 .063375 & .3329806 \\
\hline 626 & 562237.225936 .238472 .384947 & 1.688779 .148601 .189609 .516729 & 3329252 \\
\hline 628 & 620817.219815 .967674 .710332 & 1.865044 .174831 .202682 .776536 & .3328699 \\
\hline 630 & 685396.170227 .967465 .834501 & 2.059391 .647140 .527228 .529479 & .3328148 \\
\hline 632 & 756577.918078 .906296 .908312 & 2.273644 .913597 .837330 .081136 & 3327599 \\
\hline 634 & 835026.051382 .395988 .454667 & 2.509808 .051552 .031608 .082535 & .3327051 \\
\hline 636 & 921469.716245 .048299 .657364 & 2.770083 .477684 .418110 .395121 & .3326505 \\
\hline 638 & 1.016709 .984388 .014492 .641358 & 3.056891 .244979 .232231 .862474 & 3325960 \\
\hline 640 & 1.121626 .827786 .602072 .747475 & 3.372890 .185488 .482409 .685019 & .3325417 \\
\hline 642 & 1.237186 .759233 .338047 .297620 & 3.721001 .072479 .541451 .508397 & .3324876 \\
\hline
\end{tabular}




\begin{tabular}{|c|c|c|c|}
\hline$n$ & $g(n)$ & $p(n)$ & $g(n) / p(n)$ \\
\hline 644 & 1.364451 .199264 .602452 .424663 & 4.104431 .991606 .013700 .457110 & .3324336 \\
\hline 646 & 1.504585 .639672 .551644 .242118 & 4.526706 .128254 .173781 .044298 & 3323797 \\
\hline 648 & 1.658869 .675649 .684241 .358424 & 4.991692.197319.220372.390544 & .3323261 \\
\hline 650 & 1.828707 .990132 .791601 .947044 & 5.503637 .762499 .727151 .307095 & .3322725 \\
\hline 652 & 2.015642 .376610 .571304 .137220 & 6.067205 .714919 .484306 .343541 & 3322192 \\
\hline 654 & 2.221364 .899627 .572482 .931246 & 6.687514 .205661 .440172 .553650 & 3321660 \\
\hline 656 & 2.447732 .295924 .003825 .269655 & 7.370180 .353811 .425547 .662139 & .3321129 \\
\hline 658 & 2.696781 .734491 .609424 .140355 & 8.121368 .081058 .512888 .507057 & .3320600 \\
\hline 660 & 2.970748 .058134 .122963 .494038 & 8.947840 .456000 .332817 .673697 & 3320072 \\
\hline 662 & 3.272082 .647087 .407057 .382802 & 9.857016 .966290 .401433 .259592 & .3319546 \\
\hline 664 & 3.603474 .051224 .525351 .885322 & 10.857036 .174895 .938656 .583295 & .3319021 \\
\hline 666 & 3.967870 .557560 .987133 .651938 & 11.956824 .258286 .445517 .629485 & 3318498 \\
\hline 668 & 4.368504 .867675 .777863 .215047 & 13.166169 .969647 .255482 .980383 & .3317976 \\
\hline 670 & 4.808921 .083435 .523461 .447446 & 14.495806 .619536 .377005 .379418 & 3317456 \\
\hline 672 & 5.293004 .208720 .088866 .913117 & 15.957501 .720133 .631304 .230773 & .3316937 \\
\hline 674 & 5.825012 .402614 .819454 .894906 & 17.564154 .997755 .650263 .621500 & 3316420 \\
\hline 676 & 6.409612 .231875 .852908 .803564 & 19.329905 .542049 .511423 .199336 & .3315904 \\
\hline 678 & 7.051917.201670.489551.038777 & 1.270248 .929688 .765106 .878025 & .3315390 \\
\hline 680 & 7.757529 .859490 .264465 .707112 & 23.402165.235974.892374.954302 & .3314876 \\
\hline 682 & 8.532587 .803739 .101324 .785065 & 25.744258.930034.131533.263392 & .3314365 \\
\hline 684 & 9.383813 .947348 .828453 .393455 & 28.316911 .738879 .831363 .625420 & .3313854 \\
\hline 688 & 11.344923.592080.919506.168911 & 34.245325 .433219 .728719 .773420 & .3312838 \\
\hline 690 & 12.471699 .488013 .896651 .711403 & 37.652317 .810725 .762600 .765183 & .3312332 \\
\hline 692 & 13.708565 .414096 .463673 .858784 & 41.392749 .264546 .866860 .893416 & .3311827 \\
\hline 694 & 15.066103 .023547 .107434 .511935 & 45.498723 .689129 .703063 .649450 & .3311324 \\
\hline 696 & 16.555894 .604645 .352077 .748455 & 50.005385 .980149 .860746 .062163 & .3310822 \\
\hline 698 & 18.190616 .179789 .509008 .260961 & 54.951205 .445179 .608281 .719072 & .3310321 \\
\hline 700 & 19.984139 .124356 .415694 .351472 & 60.378285 .202834 .474611 .028659 & .3309822 \\
\hline 702 & 21.951641 .078449 .121951 .690536 & 66.332699 .915362 .724119 .980694 & .3309324 \\
\hline 704 & 24.109726 .981512 .974424 .928791 & 72.864864 .407855 .341219 .969825 & .3308827 \\
\hline 706 & 26.476561 .145944 .022178 .165998 & 80.029935 .953661 .656574 .123574 & .3308332 \\
\hline 708 & 29.072011 .353175 .813385 .104755 & 87.888253 .251761 .884175 .130183 & .3307838 \\
\hline 710 & 31.917806 .055993 .689642 .806479 & 96.505815 .389469 .697877 .049934 & .3307345 \\
\hline 712 & 35.037705 .854425 .086767 .315281 & 105.954804 .374756 .131323 .439197 & .3306853 \\
\hline 714 & 38.457690 .525805 .807655 .349494 & 116.314155 .138696 .524440 .183805 & .3306363 \\
\hline 716 & 42.206162.992149.595203.733066 & 127.670177 .252209 .281782 .740521 & .3305874 \\
\hline 718 & 46.314171 .740509 .991644 .623377 & 140.117232 .974725 .477106 .760252 & .3305387 \\
\hline 720 & 50.815653 .333132 .453355 .417013 & 153.758476 .658245 .881594 .406593 & .3304900 \\
\hline 722 & 55.747696 .798594 .659665 .278707 & 168.706660 .971164 .630122 .439117 & .3304415 \\
\hline 724 & 61.150831 .843648 .989160 .520517 & 185.085015 .885255 .746880 .625875 & .3303932 \\
\hline 726 & 67.069343.000364.790909.509432 & 203.028206.889569.986197.651315 & .3303449 \\
\hline 728 & 73.551612 .003580 .342261 .319587 & 222.683379 .460186 .024851 .577401 & .3302968 \\
\hline
\end{tabular}




\begin{tabular}{|c|c|c|c|}
\hline$n$ & $g(n)$ & $p(n)$ & $g(n) / p(n)$ \\
\hline 730 & 80.650490 .898600 .239564 .879402 & 244.211297 .428606 .706709 .925517 & .3302488 \\
\hline 732 & 88.423708 .591505 .850741 .335866 & 267.787583 .558210 .323920 .375877 & .3302009 \\
\hline 734 & 96.934313 .793528 .032678 .451108 & 293.604071 .362025 .285843 .562670 & .3301531 \\
\hline 736 & 106.251157 .568762 .826790 .538184 & 321.870277 .981032 .622582 .593573 & .3301055 \\
\hline 738 & 116.449418 .966522 .956877 .514932 & 352.815008 .795455 .957133 .215652 & .3300580 \\
\hline 740 & 127.611177 .530258 .752406 .622494 & 386.688105 .367749 .941220 .651375 & .3300106 \\
\hline 742 & 139.826036 .793992 .945433 .935250 & 423.762349 .321394 .151918 .928481 & .3299633 \\
\hline 744 & 153.191803 .242115 .928699 .781472 & 464.335535 .850798 .483634 .138280 & .3299161 \\
\hline 746 & 167.815225 .581219 .295311 .353031 & 508.732731 .741838 .107613 .602755 & .3298691 \\
\hline 748 & 183.812799 .611964 .924619 .350744 & 557.308734 .067567 .635805 .394638 & .3298222 \\
\hline 750 & 201.311644.414948.208412.982824 & 610.450747 .117966 .916191 .771809 & .3297754 \\
\hline 752 & 220.450456 .090175 .710516 .586817 & 668.581296 .635294 .279311 .393900 & .3297287 \\
\hline 754 & 241.380545 .790304 .317092 .400955 & 732.161402 .067670 .820574 .405230 & .3296821 \\
\hline 756 & 264.266969 .403457 .474602 .542187 & 801.694029 .333610 .862568 .750951 & .3296356 \\
\hline 758 & 289.289756 .827489 .405970 .746710 & 877.727848 .520950 .325159 .242658 & .3295893 \\
\hline 760 & 316.645249 .513934 .062456 .082785 & 960.861323 .037560 .814483 .873080 & .3295431 \\
\hline 762 & 346.547555 .632309 .591952 .786426 & 1051.747159 .001957 .690209 .588887 & .3294970 \\
\hline 764 & 379.230133 .081677 .670002 .048472 & 1151.097146 .124113 .726578 .727360 & .3294510 \\
\hline 766 & 414.947511 .367341 .686064 .123585 & 1259.687423 .996378 .387111 .229150 & .3294051 \\
\hline 768 & 453.977164.384474.119153.084260 & 1378.364210 .608578 .997366 .598385 & .3293593 \\
\hline 770 & 496.621547 .078482 .874510 .287475 & 1508.050033 .038752 .490738 .311726 & .3293137 \\
\hline 780 & 776.694909 .402394 .840837 .177170 & 2360.150221 .898687 .182164 .777966 & .3290870 \\
\hline 790 & 1211.353075 .227951 .617473 .679900 & 3683.456542 .940343 .404363 .084600 & .3288631 \\
\hline 800 & 1884.120926 .878905 .266804 .491024 & 5733.052172 .321422 .504456 .911979 & .3286418 \\
\hline 810 & 2922.713240 .872047 .520353 .037152 & 8899.229771 .588828 .461969 .917962 & .3284231 \\
\hline 820 & 4521.932323 .152782 .786266 .159359 & 13777.683783 .859651 .786576 .215682 & .3282070 \\
\hline 830 & 6978.188694 .133167 .705334 .437356 & 21275.399574 .724765 .449983 .360003 & .3279933 \\
\hline 840 & 10741.425934 .538122 .626370 .804346 & 32770.027459 .303858 .556350 .798600 & .3277820 \\
\hline 850 & 16493.048943 .715545 .894087 .350086 & 50349.216918 .401212 .177548 .479675 & .3275730 \\
\hline 860 & 25262.619707 .922527 .216551 .496044 & 77169.232591 .877674 .590168 .543277 & .3273664 \\
\hline 870 & 38602.227230 .689416 .618251 .994485 & 117991.131259 .998859 .170817 .958839 & .3271621 \\
\hline 880 & 58846.479520 .175142 .676222 .361808 & 179980.699075 .416049 .556058 .362840 & .3269599 \\
\hline 890 & 89499.347097 .873269 .012421 .364801 & 273899.386535 .208029 .575034 .561337 & .3267599 \\
\hline 900 & 135808.560233 .418108 .130691 .354490 & 415873.681190 .459054 .784114 .365430 & .3265620 \\
\hline 910 & 205616.757446 .208107 .769772 .586031 & 630018.505076 .433611 .630379 .753807 & .3263662 \\
\hline
\end{tabular}

\section{Implementation}

To implement the recursion we used a hash table to store the results computed in the recursion. This is useful as we do not need to compute all smaller results. Next trick was not to store 
smaller results, so we decided not to store results which where smaller then 400 . But independent of all the tricks the limit was the size of the memory, not the computing time. The following table shows the size of the hash table in MB together with the used CPU time in seconds on a Linux $3 \mathrm{GHz} / 4 \mathrm{~GB}$ single processor machine.

\begin{tabular}{|c|c|c|c|c|c|c|c|c|c|c|}
\hline $\mathrm{n}$ & 50 & 100 & 200 & 220 & 300 & 400 & 500 & 600 & 700 & 760 \\
\hline \hline MB & 0.04 & 1.3 & 13 & 21 & 59 & 195 & 437 & 863 & 1332 & 1889 \\
\hline \hline sec & 0 & 1 & 29 & 41 & 122 & 380 & 984 & 2009 & 3647 & 4811 \\
\hline
\end{tabular}

Above table of the number of graphical partitions up to $n=760$ was computed in a single run, where we filled the hash table with all values necessary to compute all the numbers $g(0), \ldots, g(760)$ of graphical partitions. For the values for $n>760$ we had to use a different method of computation. We computed the number of pairs $L(\lambda) \unrhd R(\lambda)$ for a given size $i$ of the Durfee square. Afterwards we remove all precomputed values $p(m, k, n, l)$ with $l<i$, and started with the next size of the Durfee square. This method works because as you see in the telescoping lemma, we need for the computation of $p(m, k, n, i)$ other values with parameter $i$ or $i-1$ only. This reduces the amount of memory necessary for the storage of precomputed values from $O\left(n^{3}\right)$ to $O\left(n^{2.5}\right)$. (thanks to the referee) This helps in the cases $m !=n$, but in the limiting case $m=n$ we have to recompute new results, but overall this trick reduces the amount of memory necessary to store intermediate results.

So we were able to compute larger values up to $n>900$. But we didn't have time to compute all the values, which explains the missing values in the above table.

A current version of the table can be fetched from

http://www.mathe2.uni-bayreuth.de/axel/numberofgraphicalpartitions.pdf.

\section{References}

[BS] Tiffany M. Barnes, Carla D. Savage, "A Recurrence for Counting Graphical Partitions", Electronic J. Comb. 2 (1995) R11, 10p.

[Ha] Werner Hässelbarth, "Die Verzweigtheit von Graphen", Habilitationsvortrag, (1983), 15p.

[Ke] Adalbert Kerber, "Applied Finite Group Actions", Springer Verlag, (1999), 454p.

[SH] Gerard Sierksma, Han Hoogeven, "Seven Criteria for Integer Sequences Being Graphic". J. Graph Th.15 (1991) 223-231. 